\title{
OBSERVATIONS PRÉLIMINAIRES SUR LE FONCTIONNEMENT DES ZONES DE FRAYĖRES DE LA TRUITE COMMUNE (SALMO TRUTTA FARIO) DANS DEUX COURS D'EAU ARIÉGEOIS (LE SALAT ET L'ALET)
}

\author{
par O. PLASSERAUD, P. LIM et A. BELAUD \\ Laboratoire d'Ichtyologie Appliquée \\ Ecole Nationale Supérieure Agronomique \\ 145, avenue de Muret - 31076 TOULOUSE Cedex
}

\section{RÉSUME}

Deux tronçons de cours d'eau ont été étudiés dans le département de l'Ariège : le Salat et son affluent I'Alet.

Les zones où le gravier avait été remanié par les truites fario lors de la période de reproduction, habituellement définies comme des frayères, ont été explorées pour vérifier la présence ou l'absence d'œufs en relation avec les géniteurs présents sur ces sites.

Les 3 et 4 décembre 1987, 43 prélèvements ont été effectués au niveau des frayères, sur des surfaces unitaires d'échantillonnage de $0,1 \mathrm{~m}^{2}$. Pour chaque point, le substrat et, le cas échéant, les œufs ont été prélevés et des mesures de vitesse et de profondeur ont été effectuées.

Les 43 unités de prélèvement ont permis de récolter 591 œufs. 25 points unités ne contenaient aucun œuf; les autres contenaient de 1 à $116 œ u f s$.

Parallèlement, 20 femelles matures, de 167 à $235 \mathrm{~mm}$, capturées par pêche électrique sur les tronçons étudiés, ont èté sacrifiées et leurs ovaires prélevés.

L'examen du nombre d'ovules présents chez les femelles lors de leur capture suggère l'existence d'un décalage de la ponte en fonction de l'âge des femelles.

Une relation croissante entre la taille des femelles et le diamètre moyen des ovules a également pu être vérifiée.

Les paramètres morphodynamiques mesurés ne permettent pas d'expliquer la présence ou l'absence d'œufs sur les frayères étudiées et diverses hypothèses sont envisagées. discutées.

La validité de la méthode d'échantillonnage et les perspectives de ce type d'étude sont

Mots-clés : Truite commune, Salmo trutta fario, frayères, œufs.

\section{PRELIMINARY SURVEY OF BROWN TROUT (SALMO TRUTTA FARIO) SPAWNING SITES}

IN TWO STREAMS OF ARIEGE (S.-W. FRANCE) : SALAT RIVER AND ALET RIVER

\section{ABSTRACT}

Two stretches of streams were studied in the French "department" of Ariège : The Salat River and its tributary, the Alet River.

The sites where gravel was reshaped by brown trout during the spawning period, usually defined as spawning sites, were investigated to check the presence or absence of eggs, related to mature females caught in the same area. sites.

On the 3rd and 4th of December 1987,43 samples $\left(0.1 \mathrm{~m}^{2}\right)$ were carried out in the spawning

For each sample, substratum and any eggs were removed, velocity and depth were measured.

591 eggs were harvested within the 43 sampling units. 25 units contained no eggs, the others showed 1 to 116 eggs.

During the sampling period, 20 mature females $(167-235 \mathrm{~mm})$, caught by electrofishing in the studied area, were sacrificed for ovary study.

The observation of the number of ovules for the sampled females suggests an imbalance in egg laying according to the age of females. 
The size of the females was proportional to the diameter of the ovules.

Morphodynamic parameters measured give no explanation of the presence or absence of eggs in the studied spawning sites and so, several hypotheses have been proposed.

The accuracy of the sampling method and prospects for those investigations are discussed.

Key-words : Brown trout, Salmo trutta fario, spawning sites, eggs.

\section{INTRODUCTION}

Alors que la reproduction artificielle de la truite est maîtrisée depuis plus d'un siècle, paradoxalement de nombreux points obscurs subsistent sur la reproduction en conditions naturelles. Les difficultés d'observation directe du comportement complexe des géniteurs lors de la ponte et l'étendue et la diversité des habitats qu'il serait nécessaire de surveiller permettent d'expliquer le caractère partiel des connaissances actuelles dans ce domaine.

Dans la pratique de la gestion des cours d'eau, une bonne compréhension du fonctionnement de la reproduction naturelle des truites est indispensable pour évaluer le recrutement de juvéniles et pour ajuster l'alevinage complémentaire.

Divers travaux ont déjà été entrepris pour mieux cerner le fonctionnement des frayères naturelles des truites fario. Ce travail vise à rechercher si, en milieu naturel, les zones de graviers remaniés par les truites lors de la période de reproduction, habituellement définies comme des frayères, correspondent automatiquement aux zones de ponte. Pour cela, une expérience de recherche des œufs sur les frayères a été réalisée sur deux rivières pyrénéennes.

\section{MATÉRIEL ET MÉTHODES}

Les observations ont été effectuées les 3 et 4 décembre 1987 dans le département de l'Ariège, sur la rivière Salat et son affluent l'Alet (fig. 1).

$\stackrel{N}{N}$

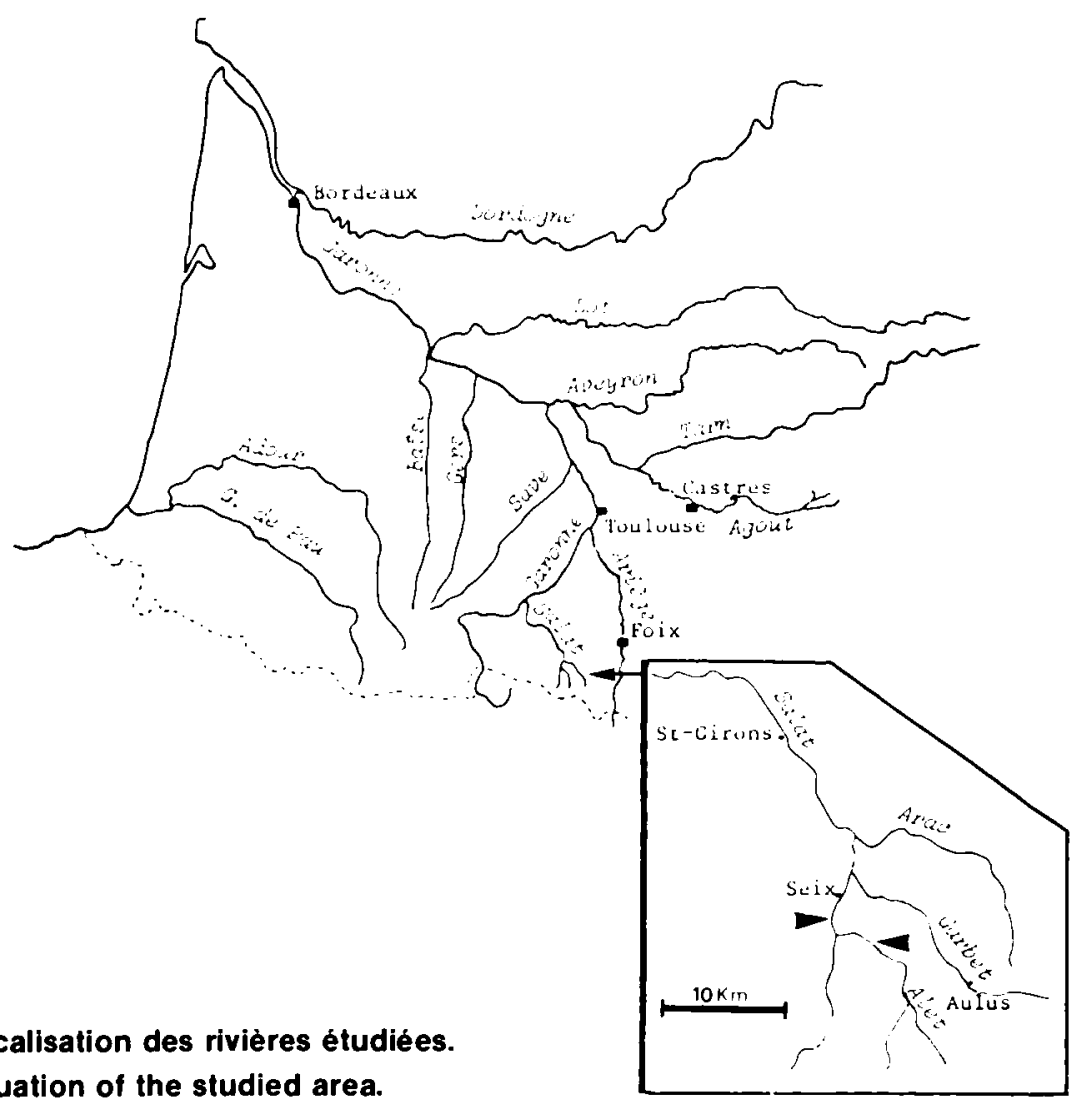


Un secteur de $400 \mathrm{~m}$ sur le Salat et un secteur de $500 \mathrm{~m}$ sur l'Alet ont été prospectés. Les caractéristiques générales des cours d'eau et des secteurs étudiés sont indiquées dans les Tableaux I et II.

Tableau I : Caractéristiques générales des cours d'eau ètudiés.

Table I : General characteristics of the studied rivers.

\begin{tabular}{|c|c|c|}
\hline Caractéristiques & Alet & Salat \\
\hline $\begin{array}{l}\text { Altitude de la source (m) } \\
\text { Longueur du cours d'eau (km) } \\
\text { Bassin versant }\left(\mathrm{km}^{2}\right) \\
\text { Confluence } \\
\text { Altitude de la confluence (m) }\end{array}$ & $\begin{array}{r}1900 \\
17 \\
90 \\
\text { SALAT } \\
560\end{array}$ & $\begin{array}{r}960 \\
21 \\
- \\
\text { ARAC } \\
450\end{array}$ \\
\hline
\end{tabular}

Tableau II : Caractéristiques générales des secteurs d'étude.

Table II : General characteristics of the studied areas.

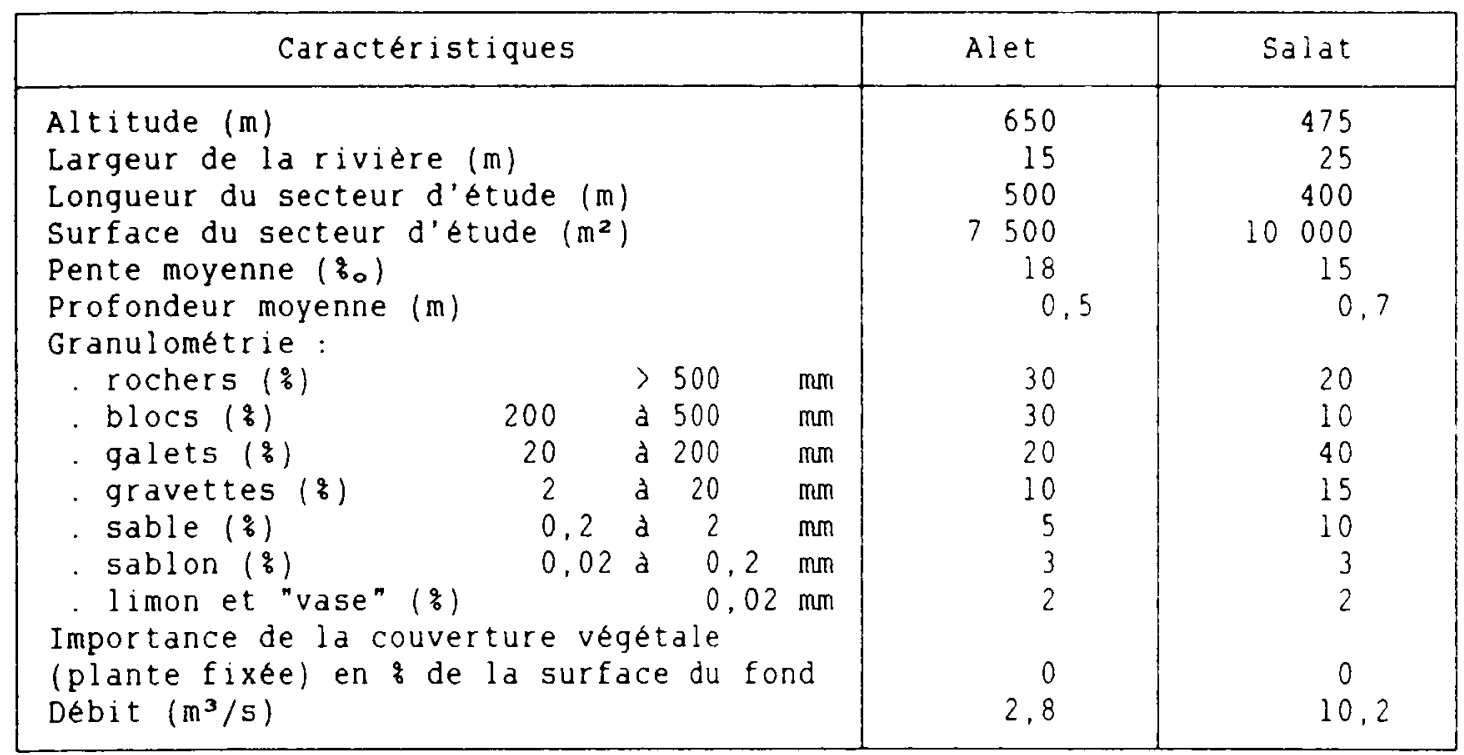

Le repérage des frayères s'est fait visuellement: les zones de graviers nettoyés et remaniés par les géniteurs, apparaissant sous forme de taches claires dans le lit des cours d'eau et présentant une partie creusée suivie d'un dôme distinct, ont été considérées comme des frayères (ALLEN, 1951). Sur le dôme des frayères ainsi définies, 43 repères (27 sur l'Alet et 16 sur le Salat) ont été installés pour matérialiser des unités de surface de $0,1 \mathrm{~m}^{2}$ (une unité de prélèvement par dôme, centrée sur le sommet de celui-ci).

Au centre de chaque unité, il a été procédé à une mesure de profondeur et trois mesures de vitesse (à $10 \mathrm{~cm}$ du fond, à mi-hauteur et en surface).

Le substrat a été prélevé en totalité dans un filet de Surber à l'aide d'une binette sur une profondeur variable selon le point de prélèvement. La profondeur fouillée a èté notée. Elle correspond à la profondeur de creusement maximale, jusqu'au contact avec un substrat compact (dalle rocheuse ou gravier colmaté à forte cohésion).

Le substrat a été trié sur une série de cinq tamis, d'un vide de maille respectivement de 37,22 , 15,10 et $2,5 \mathrm{~mm}$. Chaque fraction a été pesée. Le dernier tamis a recueilli les œufs qui ont été comptés et immédiatement placés dans une solution de conservation (acide picrique-formol-acide acétique glacial). 
Enfin, un échantillon de 20 truites femelles a été capturé par pêche électrique et sacrifié. Des écailles ont été prélevées pour connaître l'âge des géniteurs. En laboratoire, les gonades ont èté prélevées et les ovules encore présents ont été dénombrés.

\section{RÉSULTATS}

\section{- Paramètres morphodynamiques}

Les observations des caractéristiques morphodynamiques ont conduit à définir des moyennes qui apparaissent dans le Tableau III. L'écart-type, considéré comme un indice de variabilité, est aussi reporté.

\section{Tableau III : Résultats moyens des observations sur les caractéristiques morphodynamiques des $\mathbf{4 3}$ points étudiés.}

Table III : Mean results of morphodynamic measurements within 43 sampling units.

\begin{tabular}{|c|c|c|c|}
\hline Parametre & & Moyenne & Ecart-type \\
\hline Profondeur $(\mathrm{cm})$ & & 28 & 10,5 \\
\hline Vitesse $(\mathrm{cm} / \mathrm{s})$ & $\begin{array}{l}\text { surface } \\
\text { milieu } \\
\text { fond }\end{array}$ & $\begin{array}{l}44 \\
40 \\
25\end{array}$ & $\begin{array}{l}18,2 \\
16,4 \\
13,1\end{array}$ \\
\hline $\begin{array}{l}\text { Granulométrie } \\
\text { (z pondéral) }\end{array}$ & $\begin{array}{l}>37.0 \mathrm{~mm} \\
>22,0 \mathrm{~mm} \\
>15,0 \mathrm{~mm} \\
>10,0 \mathrm{~mm} \\
>2,5 \mathrm{~mm}\end{array}$ & $\begin{array}{l}30 \\
16 \\
16 \\
14 \\
24\end{array}$ & $\begin{array}{r}16 \\
6 \\
6 \\
5 \\
12\end{array}$ \\
\hline
\end{tabular}

La profondeur moyenne de la lame d'eau sous laquelle des frayères ont été observées est de $28 \mathrm{~cm}$. Le minimum est de $10 \mathrm{~cm}$, le maximum de $54 \mathrm{~cm}$.

La vitesse moyenne à $10 \mathrm{~cm}$ du fond est de $25 \mathrm{~cm} / \mathrm{s}$ (minimum $0 \mathrm{~cm} / \mathrm{s} ;$ maximum $56 \mathrm{~cm} / \mathrm{s}$ ). La vitesse moyenne à mi-profondeur est significativement $(p<0,01)$ plus élevée: $40 \mathrm{~cm} / \mathrm{s}$ (minimum $4 \mathrm{~cm} / \mathrm{s}$; maximum $77 \mathrm{~cm} / \mathrm{s}$ ) et diffère peu de la vitesse moyenne de surface : $44 \mathrm{~cm} / \mathrm{s}$ (écart-type : 18) (minimum $0 \mathrm{~cm} / \mathrm{s} ;$ maximum $79 \mathrm{~cm} / \mathrm{s}$ ).

Les graviers de gros diamètre $(>3.7 \mathrm{~cm})$ sont les plus fortement représentés dans les échantillons de substrat ( $30 \%$ du poids de l'échantillon en moyenne). Les autres fractions pondérales récoltées sont peu différentes entre elles : $16 \%(>22 \mathrm{~mm}), 16 \%(>15 \mathrm{~mm}), 14 \%(>10 \mathrm{~mm})$ à l'exception des sédiments les plus fins : $24 \%$ du poids total ont été récupérés sur le dernier tamis.

\section{- Présence d'œufs}

Le nombre d'œufs trouvés dans chaque prélèvement de substrat est indiqué dans le Tableau IV. Sur les 43 unités de prélèvement, 25 (soit 58\%) ne contenaient aucun œuf (fig. 2). 12 unités (soit $28 \%$ ) contenaient de 1 à 25 œufs. 6 unités (soit $14 \%$ ) contenaient plus de 25 œufs. Le maximum observé pour une unité de prélèvement a été de 116 œufs. 
Tableau IV : Résultats concernant la récolte des œufs sur des éléments de surtace des frayères. Table IV : Results of egg harvesting within sampling units.
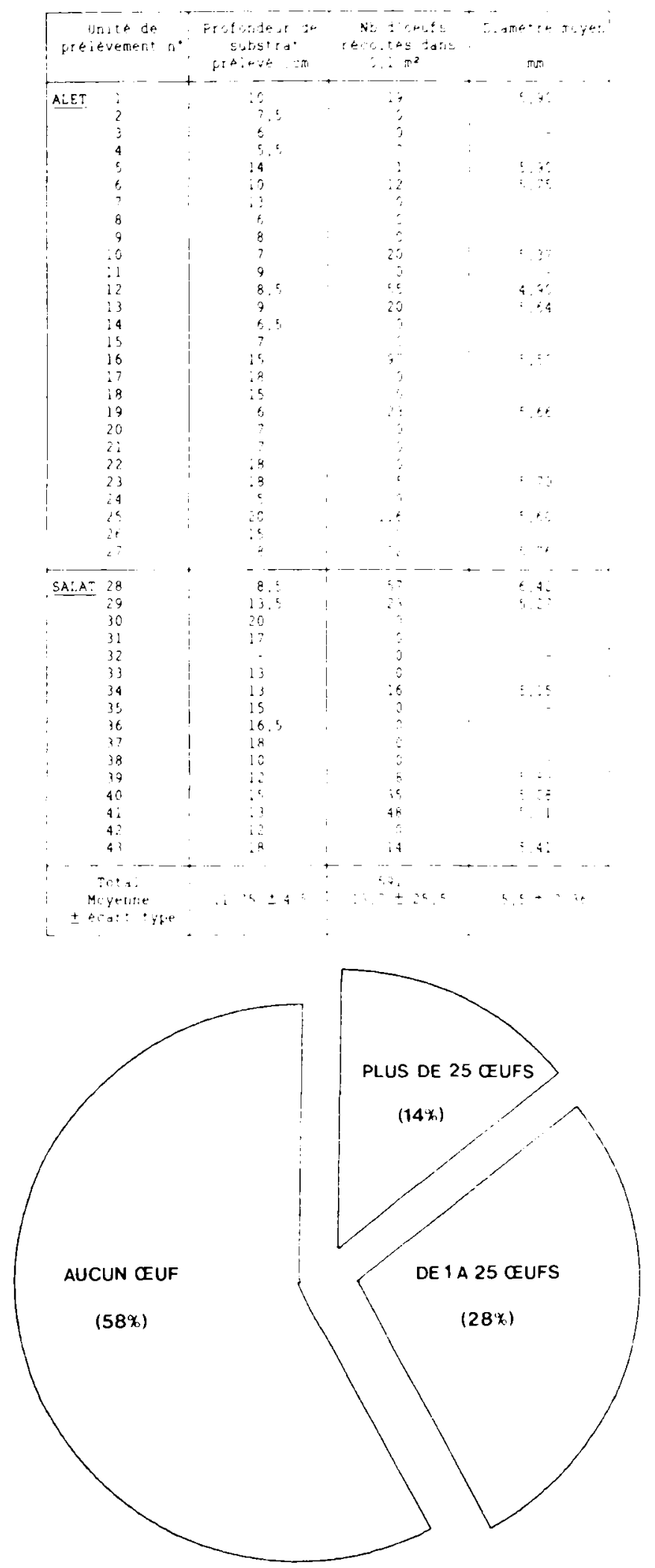

figure 2 : Classes d'abondance d'œuts par unité de prélèvement.

figure 2 : Abundance classes of eggs within sampling units. 


\section{- Géniteurs femelles capturés} Tableau $\mathrm{V}$.

Les principales caractéristiques concernant les truites fario femelles capturées figurent au

Tableau V : Principaux résultats de l'examen d'un lot de 20 femelles capturées sur le secteur des frayeres.

Table V : Main observations on a sample of 20 females caught in the studied spawning area.

\begin{tabular}{|c|c|c|c|}
\hline $\begin{array}{l}\text { Age des femelles } \\
\text { (scalimétrie) }\end{array}$ & $\begin{array}{l}\text { Longueur } \\
\text { Totale } \\
\text { (mm) }\end{array}$ & $\begin{array}{l}\text { Nombre d'ovules encore } \\
\text { présents par kilogramme } \\
\text { de femelle }\end{array}$ & $\begin{array}{l}\text { Diamètre moyen } \\
\text { des ovules } \\
\text { (écart-Type) } \\
\text { en mm }\end{array}$ \\
\hline $2^{+}$ & $\begin{array}{l}188 \\
182 \\
167 \\
178 \\
175 \\
172\end{array}$ & $\begin{array}{ll}1 & 787 \\
2 & 239 \\
2 & 172 \\
1 & 900 \\
2 & 024 \\
2 & 202\end{array}$ & $\begin{array}{ll}4,5 & (0,27) \\
3,8 & (0,25) \\
3,9 & (0,36) \\
4,3 & (0,20) \\
4,0 & (0,29) \\
3,6 & (0,33)\end{array}$ \\
\hline $\begin{array}{l}\text { moyenne } \\
\text { (écart-type) }\end{array}$ & $177(7,4)$ & $2054(182)$ & $4,0(0,33)$ \\
\hline $3^{+}$ & $\begin{array}{l}194 \\
192 \\
200 \\
203 \\
207 \\
217 \\
217 \\
214 \\
213\end{array}$ & $\begin{array}{ll}1 & 496 \\
2 & 042 \\
& 974 \\
1 & 081 \\
1 & 594 \\
1 & 961 \\
1 & 441 \\
1 & 401 \\
1 & 345\end{array}$ & $\begin{array}{ll}4,8 & (0,27) \\
4,0 & (0,31) \\
5,1 & (0,18) \\
4,4 & (0,38) \\
5,0 & (0,35) \\
5,1 & (0,20) \\
4,8 & (0,37) \\
4,9 & (0,36) \\
4,5 & (0,27)\end{array}$ \\
\hline $\begin{array}{l}\text { moyenne } \\
\text { (écart-type) }\end{array}$ & $204,1 \quad(10,1)$ & $1482(354)$ & $4,7(0,36)$ \\
\hline $4^{+}$ & $\begin{array}{l}235 \\
231 \\
235 \\
235 \\
233\end{array}$ & $\begin{array}{rr} & 10 \\
1 & 318 \\
1 & 005 \\
& 9 \\
& 59\end{array}$ & $\begin{array}{ll}4,9 & (-) \\
5,6 & (0,26) \\
4,8 & (0,27) \\
5,3 & (-) \\
5,4 & (0,27)\end{array}$ \\
\hline $\begin{array}{l}\text { moyenne } \\
\text { (écart-type) }\end{array}$ & $233,8 \quad(1,8)$ & $480(631)$ & $5,2(0,34)$ \\
\hline
\end{tabular}


Les femelles ont été réparties en trois classes d'àge pour une longueur moyenne de $177 \mathrm{~mm}$ pour les 2 ., 204, $1 \mathrm{~mm}$ pour les 3 . et $233,8 \mathrm{~mm}$ pour les 4 -. Ces longueurs sont significativement différentes d'un âge à l'autre $(p<0,01)$.

Pour les femelles de chacune de ces trois classes d'âge, le 4 décembre 1987 des ovules étaient encore présents. Ces ovules, libres dans la cavité générale et expulsables par simple pression abdominale dans le cas des femelles âgées de 3 . et 4 ., étaient en fin de maturation mais encore incomplètement détachès chez les femelles âgées de 2 .

Le nombre moyen d'ovules encore présents, rapporté au kilogramme de femelle, est significativement $(p<0,01)$ différent d'un âge à l'autre : les femelles âgées de 2 . présentaient 2.054 ovules en moyenne par kg contre 1.482 pour les femelles 3 . et 480 pour les 4 .

La mesure des diamètres d'ovules observès sur l'échantillon de femelles a permis l'établissement d'une relation sensiblement linéaire entre le diamètre moyen des ovules et la longueur de la femelle. Cette relation est représentée dans la figure 3.

Diamètre moyen des ovules $(\mathrm{mm})=0,02 \times$ longueur de la femelle $(\mathrm{mm})+0,38$

Le coefficient de corrélation est fortement significatif $(p<0,001): r=0,84$ pour $N=20$. Le diamètre moyen des ovules est significativement croissant $(p<0,05)$ d'un âge à l'autre: $4,0 \mathrm{~mm}$ pour les femelles 2 . ; $4,7 \mathrm{~mm}$ pour les femelles 3 . et $5,2 \mathrm{~mm}$ pour les femelles 4 .

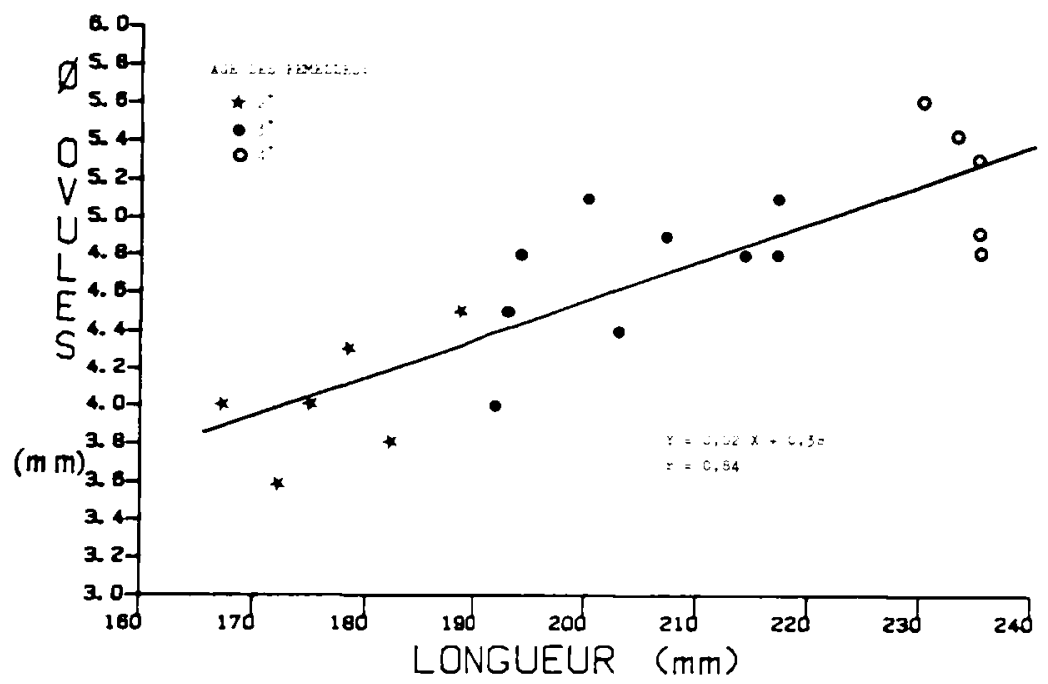

figure 3 : Diamètre moyen des ovules en fonction de la longueur des femelles.

figure 3 : Relationship between mean ova diameter and female length.

\section{DISCUSSION}

\section{- Paramètres morphodynamiques}

La profondeur moyenne de la lame d'eau sous laquelle des frayères ont été observèes $(28 \mathrm{~cm})$ est proche de l'optimum de $31,7 \mathrm{~cm}$ défini par SHIRVELL et DUNGEY (1983). La gamme observée sur l'Alet et le Salat est légèrement plus étendue (de 10 à $54 \mathrm{~cm}$ ) que celle proposée par ces auteurs (de 16,4 à $47 \mathrm{~cm}$ ).

D'autres références, récemment analysėes par FRAGNOUD (1987), mettent en évidence le fait que les frayères peuvent se trouver à des profondeurs très variables : entre $5 \mathrm{~cm}$ et $97 \mathrm{~cm}$, selon les rivières étudiées. La profondeur ne semble pas être le facteur primordial du choix de l'emplacement des frayères.

La vitesse est une caractéristique difficile à définir et les méthodologies varient selon les auteurs. La vitesse à mi-hauteur s'approche de la vitesse moyenne de l'eau sur un transect et peut caractériser l'écoulement dans son ensemble. Mais pour SHIRVELL (1988), seule la vitesse près du fond, effectivement perçue par le salmonidé sur sa frayère, serait à prendre en compte. Les présentes observations situent cette vitesse au fond à une moyenne de $25 \mathrm{~cm} / \mathrm{s}$, proche de la borne inférieure de $28,2 \mathrm{~cm} / \mathrm{s}$ définie par SHIRVELL et DUNGEY (1983). La vitesse à mi-hauteur, qui s'élève à $40,9 \mathrm{~cm} / \mathrm{s}$, est du même ordre que celle indiquée par SMITH (1973): $44,5 \mathrm{~cm} / \mathrm{s}$. 
De nombreux auteurs (STUART, 1953; HOOPER, 1973; REISER et WESCHE, 1977) ont mis en évidence la préférence de la truite fario pour des graviers de 1 à $7 \mathrm{~cm}$ de diamètre lors du choix du substrat de fraie, mais ont noté l'utilisation possible d'une gamme plus large: de 0,3 à $10 \mathrm{~cm}$ de diamètre. Nos observations se situent également dans cette classe de granulométrie mais les graviers de fort diamètre $(3,7 \mathrm{~cm})$ dominent dans les échantillons récoltés $(30 \%$ du poids en moyenne). Ceci suggère qu'ils seraient particulièrement recherchés par les truites lors de l'établissement des frayères.

Les observations concernant les paramètres morphodynamiques étudiés (profondeur, vitesse, substrat) aux 43 unités de prélèvement sur les frayères restent dans les gammes définies par les auteurs précédents. La grande variabilité de ces facteurs, selon les auteurs et, pour un même auteur, selon la zone étudiée, se retrouve dans nos observations. Les mesures effectuées ne mettent pas en évidence un créneau de préférence très étroit pour les facteurs morphodynamiques considérés.

\section{- Chronologie de la ponte}

Aucune femelle âgée de 1+ mature n' a été observée sur le Salat, alors que BAGLINIERE, LE BAIL et MAISSE (1981) ont montré que ce phénomène n'est pas rare sur le Scorff (Bretagne sud). Ceci découle vraisemblablement des faibles croissances observées : $177 \mathrm{~mm}$ en moyenne à 2. pour les femelles matures du Salat contre $186,5 \mathrm{~mm}$ à 1 + pour les femelles en vitellogénèse sur le Scorff. générale.

Les 20 femelles sacrifiées le 4 décembre 1987 présentaient encore des ovules dans leur cavité

Les ovules des truites âgées de $2+$ n'étaient pas encore expulsables par pression modérée de l'abdomen. Ceci suggère que les femelles âgées de $2+$ n'avaient pas encore frayé à cette date. Le nombre de 2.054 ovules par kilogramme de femelles observé peut donc être considéré comme une estimation de la fécondité théorique des femelles $2+$ du Salat. Cette valeur est conforme aux données habituelles (VIBERT et CUINAT, 1963). Les ovules de femelles âgées de 3 + et $4+$ étaient expulsables par simple pression abdominale et libres dans la cavité générale. La présence d'ovules en nombre moyen, assez faible chez les femelles âgées de 3+ (1.482 par $\mathrm{kg})$ et plus faible encore chez les femelles âgées de 4 + (480 par $\mathrm{kg}$ ) suggère l'existence d'une chronologie de la ponte en fonction de l'âge des femelles. Les truites les plus âgées (4+) auraient presque terminé la ponte, alors que les femelles effectuant leur première maturation $\left(2_{+}\right)$n'auraient pas encore commencé (fig. 4).

NOMBRE D'OUTLES RESTANTS

PAR KILOGRAME DE PEMELLES

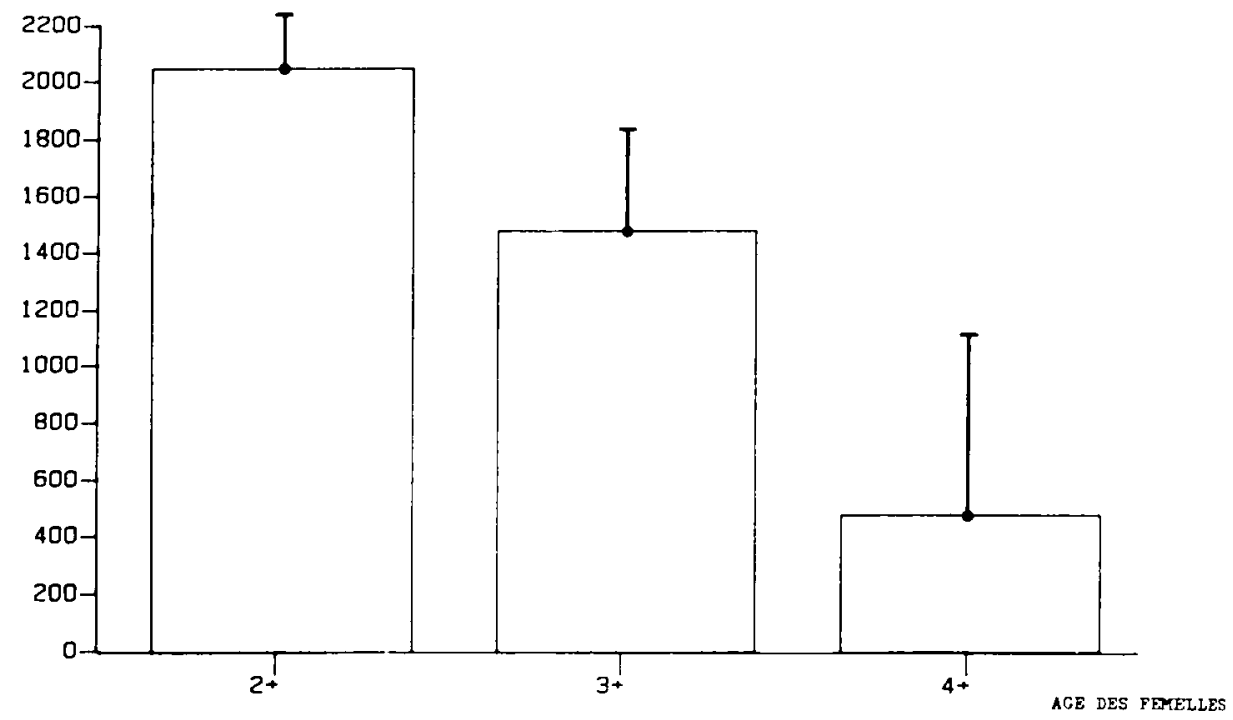

figure 4 : Nombre d'ovules encore présents par kilogramme de truites fario femelles du Salat, le 4 décembre 1987, en fonction de leur âge (les barres verticales représentent l'écart-type).

figure 4 : Number of ova still present per kilogram of female brown trouts of the Salat River, on the 4th of December 1987, according to their age (vertical bars : standard deviation). 


\section{- Cufs récoltés}

Le diamètre moyen des œufs récoltés sur les frayères est élevé : $5.5 \mathrm{~mm}$. II suggère donc également une ponte des femelles les plus âgées en raison de la relation (fig. 3) existant entre la taille de la femelle et le diamètre moyen de ses ovules.

La forte proportion de prélèvements (58\%) ne contenant aucun œuf montre que le remaniement des graviers par les truites fario dans les zones habituellement considérées comme des frayères n'est pas un critère suffisant pour conclure à la présence d'œufs.

Les résultats conduisent à émettre les hypothèses suivantes :

- la date d'observation était trop précoce et les zones remaniées mais dépourvues d'œufs correspondraient à des frayères en cours de construction ;

- un ou plusieurs facteurs défavorables perçus par les géniteurs en cours de fraie auraient arrêté le processus de reproduction après un debut de construction de frayère mais avant l'émission des gamètes ;

- l'émission des gamètes aurait eu lieu sur tout ou partie des sites dépourvus d'œufs mais les conditions morphodynamiques ou le comportement des géniteurs n'auraient pas permis l'enfouissement suffisant des cufs qui auraient alors èté balayés par le courant.

\section{- Validité de la méthode}

La surface d'échantillonnage a été fixée à $0,1 \mathrm{~m}^{2}$ sous forme d'un carré de $0,33 \mathrm{~m}$ de côté. Ce choix s'est opéré après des observations préalables ayant permis d'estimer que cette surface suffisait à couvrir entièrement le dôme de la plupart des frayères observées sur les deux cours d'eau étudiés. A l'usage, il s'est avéré que certaines zones de fraie n'étaient pas totalement recouvertes par l'appareil. Dans ce cas, la fouille systèmatique de ces zones périphériques après prélèvement de l'échantillon central n'a jamais permis d'extraire de nouveaux œufs. La surface de $0,1 \mathrm{~m}^{2}$ centrée sur le dôme de la frayère semble donc représentative d'une ponte pour le secteur d'étude considéré. Cependant, OTTAWAY et al. (1981) ont mis en évidence une relation entre la taille de la femelle et les dimensions de sa frayère. Les truites du Salat et de l'Alet sont de dimensions modestes (la plus grande femelle capturée mesurait $235 \mathrm{~mm}$ ) et l'étude des frayères de géniteurs de plus grande taille nécessiterait probablement l'utilisation d'une surface d'échantillonnage plus importante.

\section{CONCLUSION}

La présente étude suggère que les frayères de truite fario, telles qu'elles sont définies visuellement par un remaniement des graviers par les géniteurs, n'impliquent pas systématiquement la présence d'œufs.

L'étude entreprise sera complétée et approfondie afin de quantifier la ponte sur l'ensemble de la saison de reproduction suivante pour une portion de cours d'eau au peuplement connu. Une telle approche devrait permettre une meilleure connaissance des potentialités de la reproduction naturelle dont les méthodes d'estimation actuelles, par l'intermédiaire de la fécondité théorique ou le comptage visuel des frayères, ne permettent pas d'évaluer la quantité d'œufs réellement susceptibles de jouer un rôle dans le recrutement d'une population naturelle.

\section{REMERCIEMENTS}

Les auteurs tiennent à exprimer leurs très sincères remerciements à Monsieur J. DELQUE, Président et Monsieur G. CATHARY, garde-chef principal de la Fédération des Associations de Pêche et de Pisciculture de l'Ariège, ainsi qu'à Monsieur C. TERTRE. Président de l'Association du Cabilhat du canton d'Oust, pour l'aide apportée à la réalisation de ce travail.

\section{BIBLIOGRAPHIE}

ALLEN K.R., 1951. The Horokiwi stream, a study of a trout population. New-Zealand Marine Dept. Fish. Bull., 10, 231 p.

BAGLINIERE J.M., LE BAIL P.Y., MAISSE G., 1981. Détection des femelles de salmonidés en vitellogénèse. 2. Un exemple d'application : recensement dans la population de truite commune (Salmo trutta) d'une rivière de Bretagne sud (le Scorff). Bull. Fr. Piscic., 283 : 89-95.

FRAGNOUD E, 1987. Préférences d'habitat de la truite fario (Salmo trutta fario) en rivière. Thèse de $3^{\text {e }}$ cycle, Université Claude Bernard Lyon, $435 p$.

HOOPER D.R., 1973. Evaluation of the effects of flows on trout stream ecology. Pacific Gas and Electric Company, Dept. Eng. Res., Emeryville, CA, 97 p.

OTTAWAY E.M., CARLING P.A., CLARKE A., READER N.A., 1981. Observations on the structure of trout, Salmo trutta L., redds. J. Fish Biol., 19, 135-145. 
REISER D.W., WESCHE T.A., 1977. Determination of physical and hydraulic preferences of brown and brook trout in the selection of spawning locations. Water Ressource Res. Ser. $n^{\circ} 64$, $100 \mathrm{p}$.

SHIRVELL C.S., DUNGEYR.G., 1983. Microhabitat chosen by brown trout for feeding and spawning in rivers. Trans. Am. Fish. Soc., 112 (3), 355-366.

SHIRVELL C.S., 1988. Ability of PHABSIM to predict chinook Salmon spawning habitat. Fourth International Symposium on Regulated Streams, Loughborough, 1988.

SMITH A.K., 1973. Development and application of spawning velocity and depth criteria for Oregon salmonids. Trans. Am. Fish. Soc., 102, 312-316.

STUART T.A., 1953. Spawning migration, reproduction and young stages of Loch trout (Salmo trutta L.). Freshwater and Salmon Fish. Res., 5, 81 p.

VIBERT R., CUINAT R., 1963. Diagnose démographique dans les populations de poissons des cours d'eau à truites. Stud. Rev. Gen. Fish. Conc. Médit., $n^{\circ} 21$. 